\section{ED overcrowding}

To the Editor: Overcrowding is a major international problem that compromises clinical and educational activities in the emergency department. I have spent some interesting time over the last few days. I have visited 9 accident \& emergency (A\&E) departments in North London, England, to assess their capacity to train Specialist Registrars in Emergency Medicine on behalf of the Joint Committee on Higher Specialist Training in A\&E Medicine.

Two years ago all 9 of these departments had severe overcrowding with all the ensuing difficulties that arise from clinical incidents to increased staff sickness and poor morale. Since then, the government has made emergency care a priority and the UK National Health Service (NHS) has introduced a 4-hr rule: Everyone attending an A\&E department, both discharges and admitted patients, must have left within 4 hours of arrival. Now, none of the departments I visited had overcrowding. The waiting rooms were largely empty, and many departments had plenty of available treatment spaces. These changes have come about largely because of improvements in whole-system process issues and an increasing focus on activities outside the A\&E department.

Increased funding has been made available for extra medical and nursing staff, and a wide variety of clinical decision unit models have evolved, staffed in some cases by consultants in A\&E medicine and sometimes by "Acute Care Physicians." There has been no huge increase in bed numberss, yet capacity has improved: The hospital emphasis appears to have shifted to discharge rather than admission. While many have spoken softly, a big stick has been wielded. Accountability for "breaches" in the 4-hr rule lie with the CEO of the hospital, and these breaches have become a "sackable" offence. This attitude has also diffused through to inpatient wards, where undeclared vacant beds are equally a disciplinary offence for staff. Self preservation has motivated management to ensure that beds are declared available at the earliest opportunity, discharge lounges have been established in all hospitals, community support has improved to accentuate early discharge, and "discharge teams" are prevalent.

However the pressure to meet " 4 -hr targets" has brought some difficulties: Allegations of bullying and harassment by management are not uncommon, and the emphasis on service has distracted from training in some cases. Nevertheless, these departments are unrecognizable from a few years ago. Furthermore, this dramatic reduction in A\&E department overcrowding has occurred despite an increase in department attendances of at least $15 \%$ nationally in the last year (partly because of changing general practitioner availability and partly because A\&E departments are victims of their own success).

Thought you all might be interested.

\section{John Ryan \\ Consultant in Emergency Medicine \\ St. Vincent's University Hospital \\ Dublin, Ireland}

\section{ESI and CTAS}

To the Editor: We read with interest Grafstein's commentary ${ }^{1}$ on Worster and colleagues' article, ${ }^{2}$ "Assessment of inter-observer reliability of two fivelevel triage and acuity scales: a randomized controlled trial" in CJEM's July 2004 issue.

Worster and colleagues sought to compare the inter-observer reliability of
ESI [Emergency Severity Index] and CTAS [Canadian Emergency Department Triage and Acuity Scale] using weighted quadratic kappas. Grafstein disagreed with the use of weighted quadratic kappas and stated that "agreement is agreement" and that "weighted kappa scores tend to overestimate the level of agreement between observers." He suggests that "unweighted kappa value and the raw agreement on exact triage level" as well as "unweighted kappa values between adjacent triage levels" be reported.

According to statistical principles, reliability in this instance is a measure of the extent to which a triage scale gives the same acuity level over different situations (e.g., different observers or days) and a measure of the extent to which one can differentiate among patients on acuity levels. This is calculated as the true variance among the patient acuity divided by the sum of the true variance and the error variance divided by the number of levels in the triage scale. Inter-observer reliability specifically examines the degree of agreement of the acuity levels between different observers using the same triage scale. ${ }^{3}$ One way of expressing inter-observer reliablity is with weighted or unweighted kappa levels. Using these principles, there are 3 flaws to Grafstein's commentary.

Firstly, "agreement is [only] agreement" when one can convince oneself that a true Level 2/II (ESI/CTAS) being mis-designated as a Level $3 /$ III versus a Level $5 / \mathrm{V}$ has the same clinical implications. The purpose of weighting is to evaluate the degree of disagreement of triage levels between different observers. Therefore, a weighted kappa more closely reflects the clinical implications of disagreements among triage levels, whereas unweighted kappas do not.

Secondly, triage reliability as de- 